\title{
Efficient Resource Utilization in Virtual Cloud Computing Environment
}

\author{
Akshay Kumar Gupta \\ DIT University, \\ Dehradun, Uttarakhand \\ India
}

\author{
Pradeep S. Rawat \\ DIT University, \\ Dehradun, Uttarakhand \\ India
}

\begin{abstract}
Cloud computing is a platform that provides user to implement revolutionary technologies. The main phenomena of cloud computing is based on accessing the resources using remote computation. Task scheduling is one of the major area that should be focused on. In cloud environment there may be a condition where the resources are limited that may affect resource availability. This paper presents an Enhanced version of MaxMin task scheduling algorithm that improves the turnaround time. The tasks are divided in two groups and the larger task is assigned to the resource (virtual machine) with high mips rate and other task is assigned to resource with low mips rate. To perform the experiment CloudSim toolkit is used. Our result shows that the Enhanced MaxMin algorithm gives the better result.
\end{abstract}

\section{General Terms}

Cloud Computing, CloudSim, Simulation and Modeling, Scheduling Algorithms

\section{Keywords}

CloudSim, MaxMin, Cloud computing, task scheduling, virtual machine, resource utilization, performance

\section{INTRODUCTION}

Cloud computing is the technology to provide broad access to the IT services over Internet. This is a type of distributed computing. Resource management is the essential task in cloud computing to provide reliable processing of the services. The main services provided by the cloud computing is hardware and software by the cloud service provider on the basis of pay per use. Service provider contains storage and computing server or blade server. A distributed environment is created while combining all of these recourses. Cloud Computing environment is dynamic in nature which raise the drawback that can be overcome by deploying a scheduling mechanism to ensure the proper utilization of the computing nodes. Scheduling is the technique to map the task or cloudlets to the targeted virtual machine (VM) to increase the resource utilization. Simulation tools are needed to control this type of distributed system, which are based on objectoriented programming. Some of the tools are CloudSim, Simjava, CloudAnalyst etc. that can give the idea about deploying application and understating the IT requirements. Information about cloud recourses can be provided by simulation tool. Simulation tools are strongly powerful as one can even setup different cloud configuration with Internet characteristics. It provides the facility to generate your own policy.

The main purpose of this research paper is to compare the cloudlet scheduling algorithm to find the best algorithm used for faster execution of tasks. Cloudlets are small scale datacenter which contains various resources. Cloudlets are used to get the higher throughput by avoiding network latency while accessing Cloud datacenter. CloudSim simulation tool has been used to perform comparison between various scheduling algorithms. CloudSim provides a framework that is generalised and extensible which enables seamless modeling and simulation for performance analysis.

In this paper Enhanced MaxMin algorithm is introduced to reduce the turnaround time. The paper is organised as follows: Section 1 provides the introduction to the topic. Section 2 explains the related work that was studied for this topic. Section 3 provides the explanation of the toolkit used. Section 4 gives the problem description. Section 5 tells about simulation configuration parameters. Section 6 provides the experiments and results. Section 7 gives the final conclusion. Section 8 contains the acknowledgement and Section 9 contains the used references.

\section{RELATED WORK}

Cloud computing provides massive distributed environment. it becomes very tough to control the performance evaluation due to resources and users are distributed in multiple different locations using their own policies. They simulated a Grid environment to demonstrate suitability of GridSim toolkit. They deployed Gridlet on grid resources. Resources include machine properties such as MIPS rating. In this Simulation process, resource entities and network topology is used in GridSim. The obtained results are for different grid resource, which are distributed over globe. Due to this they were able to evaluate the performance and scalability of their scheduling policies with different grid configuration such as cost, capability, etc. They concluded that the results obtained are promising [1]. In parallel and distributed environment, it is very hard to achieve high performance. The purpose of scheduling is to find a mapping of the task onto the processor and also execution of the tasks in feasible order. In [2] they have proposed a new task scheduling algorithm that is the enhanced version of MaxMin algorithm. A Workflowsim toolkit has been used to perform the experiment for dependent tasks. Cloud computing has its core component which is virtualization technology. It provides an effective way to manage the dynamic resources on cloud computing platform. In [3] they used a scheduling strategy using load balancing of VM resources based on genetic algorithm computes the total influence on system after the deployment of the required VM resources and the chose the least-affective solution for best load balancing. their results show that the proposed method can perform better load balancing and proper utilization. Cloud computing is recently got a rapid growth in the domain of information technology. Technology is in development mode mainly in resource management and task scheduling. In [4], they modified the genetic algorithm where each processor 
's processing power was different. they computed the completion time of cloudlets. Each processor is assigned varying processing power and respective cost in Indian rupees. Make-span (completion time of cloudlets) is computed and the corresponding cost of output schedules of above two algorithms and compared them. Their experimentation methodologies need the simulation tool like CloudSim. Experiment results show that under heavy loads their proposed algorithm, which was modified from genetic algorithm presents a better performance. In [5], Statistical data for the social networking application, and configure the simulation scenarios and identifying the best cloud configuration for actual deployment application. Deploying application in cloud platform with minimum cost needs experimentation methodologies that include simulation tools like CloudSim, GridSim etc.

\section{CLOUDSIM}

CloudSim is a extensible simulation toolkit that supports modelling and simulation for cloud computing system and provisioning environment.

CloudSim toolkit supports various component like configuring datacenter, Virtual machine (VMs) and defining resource provisioning policies[6]. It provides modelling and simulation at all levels of cloud computing environment.These are PaaS (Platform as a Service), SaaS (Software as a Service) and IaaS (Infrastructure as a Service).

CLOUDSIM: A TOOLKIT

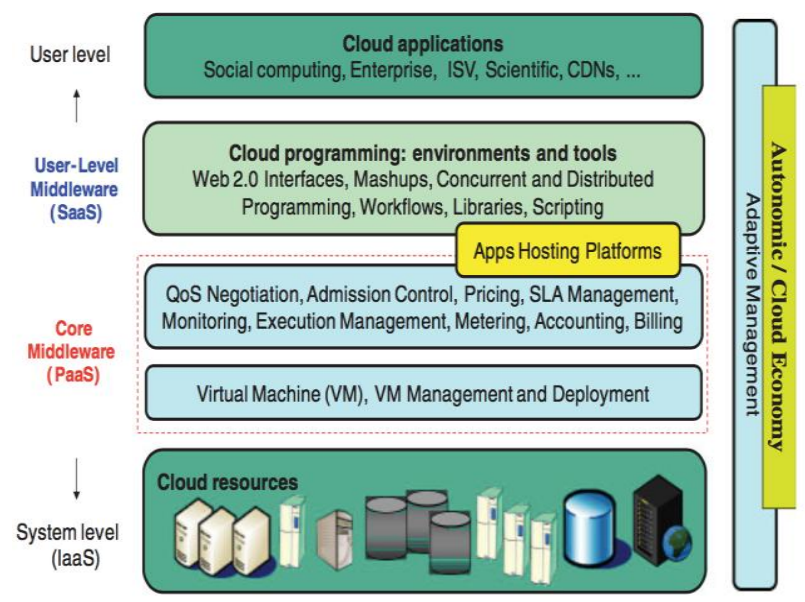

Fig 1: CloudSim Toolkit Architecture

CloudSim toolkit is based on Java and can be integrated with eclipse and NetBeans IDE. User can simulate the cloud environment using CloudSim with basic Java programming.

\section{PROBLEM DESCRIPTION}

Many traditional algorithms are available for task scheduling but they have some disadvantages in scheduling. These algorithms also have some advantages; these advantages can be used to improve the traditional algorithms. FCFS provides the task scheduling that does not schedule according to cloudlet's properties like total execution time of particular cloudlet. MaxMin provides a better result with the comparison of other traditional algorithm. But the disadvantage of the MaxMin is, if a bigger task is assigned to the slower mips resource then it may cause expansion of total turnaround time. To remove this disadvantage, the Enhanced version of MaxMin performs better than other discussed algorithm.

\section{SIMULATION CONFIGURATION PARAMETERS}

To setup the application these configurations are required:-

Table 1. Application Deployment Parameters

\begin{tabular}{|l|l|l|l|l|}
\hline $\begin{array}{l}\text { Data } \\
\text { center }\end{array}$ & $\begin{array}{l}\text { No. } \\
\text { of } \\
\text { VM }\end{array}$ & $\begin{array}{l}\text { Image } \\
\text { Size } \\
\text { (Mb) }\end{array}$ & $\begin{array}{l}\text { Memory } \\
\text { (Mb) }\end{array}$ & $\begin{array}{l}\text { Band } \\
\text { width } \\
\text { (Mbps) }\end{array}$ \\
\hline DC1 & 2 & 100000 & 1024 & 1000 \\
\hline
\end{tabular}

Table 1 shows the datacenter characteristic that contains two VMs. Details of VMs is given in table 2.

Table 2. VMs details

\begin{tabular}{|l|r|r|r|}
\hline VM & MIPS & $\begin{array}{l}\text { Image } \\
\text { size }\end{array}$ & \multicolumn{2}{|l|}{ RAM } \\
\hline VM1 & 250 & 10000 & 512 \\
\hline VM2 & 500 & 10000 & 512 \\
\hline
\end{tabular}

Table 2 contains the details of VMs. VM1 has the lower speed than VM2 with other same VM parameters.

Table 3. Host details for Datacenter

\begin{tabular}{|l|l|l|l|c|}
\hline I.D. & $\begin{array}{l}\text { Memory } \\
\text { (Mb) }\end{array}$ & $\begin{array}{l}\text { Band } \\
\text { Width } \\
\text { (Mbps) }\end{array}$ & $\begin{array}{l}\text { Vm } \\
\text { policy }\end{array}$ & $\begin{array}{l}\text { Process } \\
\text { or speed } \\
\text { (MIPS) }\end{array}$ \\
\hline 0 & 2048 & 1000000 & $\begin{array}{l}\text { Space } \\
\text { shared }\end{array}$ & 10000 \\
\hline
\end{tabular}

\section{EXPERIMENTS AND RESULTS}

Overcoming the limitation of existing algorithm does the enhancement of the MaxMin algorithm. FCFS, MinMax, MaxMin, MaxMax and MaxMin enhanced algorithm are applied.

Simulation results obtained by using CloudSim are shown below in the tabular form. These simulation results are helpful for the performance analysis of cloud based application. Different scenarios are used for the Cloud model with variable parameters with simulation results.

Table 4: Total finish time of scheduling algorithms (in sec)

\begin{tabular}{|c|c|c|}
\hline Algorithms & $\begin{array}{c}\mathbf{1 0} \\
\text { Cloudlets }\end{array}$ & 100 Cloudlets \\
\hline FCFS & 97 & 2125 \\
\hline MinMax & 135.31 & 2725 \\
\hline MaxMin & 67.5 & 1475 \\
\hline MaxMax & 74.6 & 2075 \\
\hline MaxMin_Enh & 60.9 & 1433 \\
\hline
\end{tabular}



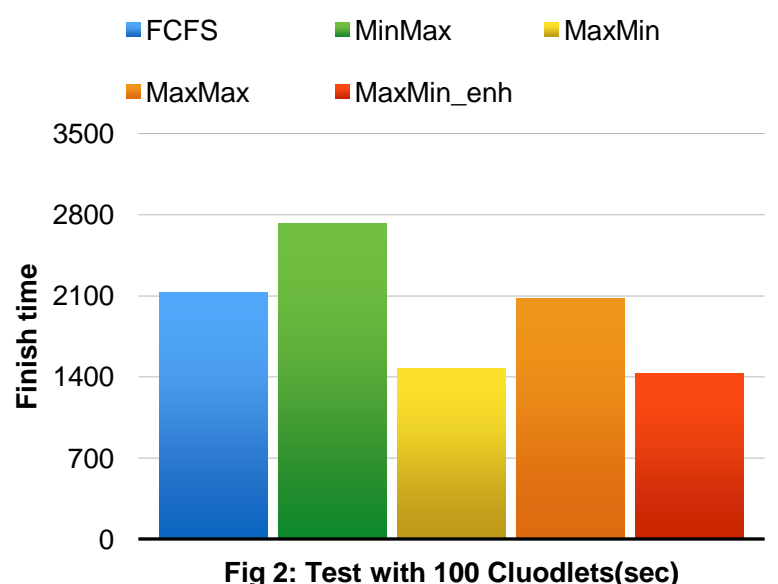

Fig 2: Test with 100 Cluodlets(sec)

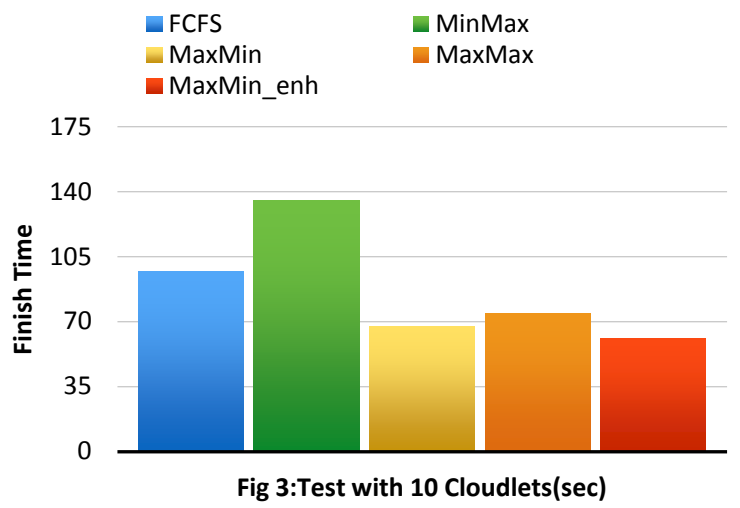

This comparison table is obtained by testing the algorithms with the set of independent 10 cloudlets and 100 cloudlets. Table 1 shows the result of performed experiment. A graphical representation of the result is shown in Fig 2 and Fig 3. The result shows that the Enhanced MaxMin algorithm executes the cloudlets with less finish time. Enhanced MaxMin algorithm can improve the performance with respect to finish time.

\section{CONCLUSION}

The comparison result shows that enhanced MaxMin and MaxMin algorithms gives the better results than other algorithms. MaxMin algorithm also provides good performance, but in MaxMin algorithm the task is divided into two groups, one group consists of the tasks that are of larger length and other group consists of the tasks that are of smaller length. In enhanced MaxMin algorithm two groups are divided according to the total average length of the tasks. If the length is higher than the average length, then it is executed on a resource with higher mips rate and if the length is smaller than the average length, then it is executed on a resource with lower mips rate. So conclusion is the enhanced MaxMin algorithm gives the better result.

\section{ACKNOWLEDGMENT}

I thank Mr. Pradeep Singh Rawat, Assistant Professor (DIT University, Dehradun, INDIA) who guided me through my research-work.

\section{REFERENCES}

[1] Rawat, Pradeep Singh, Anuj Kumar Yadav, and Varun Barthwal. "Grid resource computing environment simulation using GridSim toolkit." Computing for Sustainable Global Development (INDIACom), 2015 2nd International Conference on. IEEE, 2015.

[2] Santhosh, B., and D. H. Manjaiah. "A hybrid AvgTaskMin and Max-Min algorithm for scheduling tasks in cloud computing." Control, Instrumentation, Communication and Computational Technologies (ICCICCT), 2015 International Conference on. IEEE, 2015.

[3] $\mathrm{Hu}$, Jinhua, et al. "A scheduling strategy on load balancing of virtual machine resources in cloud computing environment." Parallel Architectures, Algorithms and Programming (PAAP), 2010 Third International Symposium on. IEEE, 2010.

[4] Kaur, Shaminder, and Amandeep Verma. "An efficient approach to genetic algorithm for task scheduling in cloud computing environment." International Journal of Information Technology and Computer Science (IJITCS) 4.10 (2012): 74.

[5] Rawat, Pradeep Singh et al. "Performance analysis of cloud computing environment and comparison with IaaS model using cloud analyst" Advances in Electronics Electrical and Computer Science Engineering, 2012.

[6] Calheiros, Rodrigo N., et al. "CloudSim: a toolkit for modeling and simulation of cloud computing environments and evaluation of resource provisioning algorithms." Software: Practice and experience 41.1 (2011): 23-50. 